\title{
THE SPACE, LUMINOSITY AND VELOCITY DISTRIBUTION OF FAINT STARS
}

\author{
William F. van Altena ${ }^{a}$, John T. Lee, Wean-Shun Tsayb) \\ Yale University Observatory \\ New Haven, CT 06511
}

\author{
Phillip K. Lua) \\ Department of Physics and Astronomy \\ Western Connecticut State University \\ Danbury, CT 06810
}

\section{INTRODUCTION}

The objective of this investigation is the determination of absolute parallaxes and proper motions with respect to faint galaxies for three fields at mid galactic latitudes and one field near the south galactic pole. The three mid galactic latitude fields were photographed on a IIIa-J + GG385 plate/filter combination by van Altena at the prime focus of the KPNO 4-meter in the mid 1970s, while the south polar region was photographed on a IIIa-F + RG610 plate/filter combination by van Altena and C.E. Lopez (Univ. of San Juan, Argentina) at the prime focus of the CTIO 4-meter; all plates were hypersensitized by baking and exposed to the sky limit. In this paper we report on the progress of an investigation of the mid galactic latitude field at R.A. $=7^{\mathrm{h}} 07^{\mathrm{m}} 31^{\mathrm{s}}$ and Dec. $=+7^{\circ} 19.7^{\prime}$ (1950) which contains the faint binary white dwarf LP543-32/33. Preliminary measurements of selected stars in this field were made some time ago and a parallax of the white dwarf binary were published by van Altena and Mora (1977). Those measurements showed that the plates were of very high astrometric quality and that it would be possible to obtain accurate parallaxes for very faint stars.

\section{ASTROMETRY}

This first field has now been completely measured by Tsay on the Yale PDS with the laser interferometer measuring system (J.-F. Lee, et al, 1986) which provides a relative positional accuracy of approximately 0.3 microns. A total of some 7000 objects were catalogued on the best plate using a program written by A. Oemler. This large scan catalogue was then broken down into five separate catalogues with two hundred common stars so that the scan times would be shorter, since we had been experiencing failures with the system on very long scans. These failure problems have now been eliminated with the replacement of the DCRS interface with a new module designed and constructed in the Yale Center for Electronic Services by A. Wandersee. To monitor and correct for thermal drift in the system, ten well formed drift stars distributed over the whole plate were selected for

a) Visiting Astronomer, Kitt Peak National Observatory, National Optical Astronomy Observatory, which is operated by the Association of Universities for Research in Astronomy, Inc., under contract with the National Science Foundation.

b) Currently at the Center for High Angular Resolution Astronomy, Georgia State University, Atlanta, GA. 
measurement after every 100 objects in the catalogue. These final catalogues were then scanned on all 22 plates of the region.

The astrometric image positions were calculated using the Yale Digital Image Centering algorithms as outlined by J.-F. Lee and van Altena (1983). These algorithms fit the measured image density array to a symmetric Bivariate Gaussian model. Using the parameters of the model fit to each image, it is then possible to form image shape parameters that enable one to distinguish between uncrowded stars, blends of images and galaxies. We have used such image shape parameters to separate the stars from the galaxies for the later analyses. The mean positions of the drift stars in each of the scans were then plotted versus time and an interpolation curve used to correct the individual measures back to the beginning of each scan. Having corrected for the thermal drift, the five separate scans of each plate were then combined into one using a linear least squares solution and the two hundred stars common to all five scans. The errors introduced in the correction for thermal drift and the combination of the five scans are probably less than the measuring error of 0.3 microns.

Since the prime focus field of the 4-meter telescope has very strong field distortion, the plate scale changes with distance from the plate center; it was therefore necessary to correct for the distortion before the parallax solutions. Unfortunately, we do not have a flat field reference frame available, therefore we have used Chiu's (1976) investigation of the distortion parameters in several reflectors, including the KPNO 4-meter, to provide the correction to a "flat-field". All of the measured objects were then corrected by Chiu's distortion parameters to form our final list of coordinates to be analyzed for parallax and proper motion.

A selection of about four hundred reference stars uniformly distributed over the plate and in magnitude was then made for the determination of the plate constants, parallaxes and proper motions using the Yale programs described by van Altena, et al (1986). The solutions are iterative in nature, and solve first for the plate constants and then for the parallaxes and proper motions; convergence is generally found after 3 - 6 iterations. The plate constant solutions included linear and quadratic terms in the coordinates, the latter being required to allow for changes in the distortion parameters from plate to plate. Magnitude terms were initially included to gauge the ultimate accuracy of the solutions but later deleted, since they remove a part of the secular parallax that we are interested in determining. It soon became obvious that those stars close to the plate limit of our best plate, which had been used to create our initial measuring catalogue, were missing on many of the other plates and did not have high enough signal-to-noise to yield good positions. Those poorer images were therefore deleted from further analysis resulting in our final list of 5102 stars and 55 galaxies. In addition, we deleted five of the plates which were taken in rather poor seeing, since it was found that their accuracy was significantly lower than that of the other 17 plates taken in good seeing.

A plot of the parallax errors as a function of distance from the optical axis shows that the majority of the stars within 25 arc-min of the optical axis have parallax errors of 5 mas (s.e.), where 1 milli-arc-sec (mas) $=0.001$ arc-sec. Beyond this distance the reference frame is not adequate and the accuracy of the parallaxes and proper motions degrades rapidly. The frequency distribution of the parallaxes in different magnitude groupings shows that the average error for stars with $\mathrm{m} \leq 19-20$ is $\approx 3$ mas, while the error for the fainter stars is $\approx 7$ mas. A further analysis of the astrometric data awaits the completion of the photometric calibration. 


\section{PHOTOMETRY}

CCD photometry in the BVR, GG385 and GG475 systems were obtained by Lu with the \#1 0.9 meter telescope at KPNO. The BVR standards were selected from the Landolt (1983) list of standard stars. To match our blue IIIa-J + GG385 plate/filter combination, a standard GG385 + liquid CuSO4 $(2 \mathrm{~mm})$ filter was used, while for the still to be obtained red plates (IIIa-F + GG495) a GG475 with an interference coating defining the red cutoff was used. The peak transmission of the blue filter system is $87 \%$, with $60 \%$ points at 3900 and $5500 \AA$ and a FWHM range of 3750 to $5750 \AA$. The peak transmission of the red filter is about $90 \%$ with FWHM points at 4800 and $7200 \AA$.

In order to calibrate the radial photometric effects on our plates, eight $\mathrm{CCD}$ calibration regions evenly distributed in radius were selected. The exposure times used ranged from 10 seconds for the Landolt standards of the 13th magnitude, to 40 minutes for a limiting magnitude of 21 . The photometric calibrations were carried out with the AED image processing system by Lee and Lu using the DAOPHOT programs written by Stetson (1987).

We are now in the process of cross-corellating the photometric and astrometric star lists, after which the calibration of the PDS photometric photometry can be completed. The final step will be the analysis of the combined photometric and astrometric data to determine the spatial, luminosity and kinematic characteristics of the stars in this region of the sky.

\section{ACKNOWLEDGEMENTS}

We would like to acknowledge the assistance and guidance of a number of individuals who have helped us during the course of this investigation: Dr. A. A. Hoag and Mr. W. E. Schoening provided encouragement and helpful advice during the photographic observations with the 4-meter at KPNO; Drs. A. Oemler and G. DaCosta instructed us in the use of the PDS star/galaxy finding program and DAOPHOT, respectively; Dr. R. Probst helped to select the filter combinations for our photoelectric observations at KPNO; Mr. W. Lund, Director of the Yale Center for Electronic Services, and his associates Mr. L. Berman and Mr. A. Wandersee have been responsible for upgrading, maintaining and turning the PDS microdensitometer into a precision measuring machine. Finally, we would like to thank the National Science Foundation, for providing funds for the purchase of the PDS, computational equipment and research support for the investigations throughout the course of this work.

\section{REFERENCES}

Chiu, L.-T.G.: 1976, Publ. Astron. Soc. Pacific 88, 803.

Landolt, A.U.: 1983, Astron. J. 88, 439.

Lee, J.-F., Tsay, W.-S., and van Altena, W.F.: 1986, IAU Symposium No. 109, "Astrometric Techniques" , H.K. Eichhorn and R.J. Leacock, eds., D. Reidel, Dordrecht, p. 237.

Lee, J.-F., and van Altena, W.F.: 1983, Astron. J. 88, 1683.

Stetson, P.B.: 1987, Publ. Astron. Soc. Pacific 99, 191.

van Altena, W.F., Auer, L.H., Mora, C.L., and Vilkki, E.U.: 1986, Astron. J. 91, 1451. van Altena, W.F., and Mora, C.L.: 1978, Bull. Amer. Astron. Soc. 9, 599. 